\title{
INFLUENCIA DE LA FAMILIA EN EL DESARROLLO DE PAUTAS INADECUADAS DE CONDUCTA
}

\author{
ANTONIO JOSE BELLIDO ALONSO y ESTHER VILLEGAS \\ CASTRILLO
}

Profs. del Area de TS y SS.SS, EUTS de Alicante

\section{EL PUNTO DE PARTIDA}

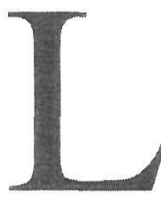

a influencia de la familia en el desarrollo de la personalidad constituye un hecho ampliamente reconocido y estudiado por las diversas disciplinas que analizan y valoran la conducta humana. El hecho de que esta influencia pueda degenerar en modos de comportamiento que son expresión de un desarrollo inadecuado de los individuos y lleva consigo una disfunción evidente de sus roles específicos es, sin duda, un problema que han de afrontar cotidianamente los Trabajadores Sociales y que vamos a abordar, aquí y ahora, teniendo presente de modo particular la incidencia que tiene en relación con los menores.

El punto de partida va a ser la referencia a una experiencia personal que hemos vivido coincidiendo con los comienzos de nuestra actividad profesional. Se trataba de un niño de dos años, que formaba parte de una familia considerada por nosotros como un ejemplo típico de la llamada «familia multiproblema». En las sucesivas visitas a su domicilio, la madre manifestó en una ocasión que el hecho de que siempre estuviera sentado en su carrito se debía a que de este modo no constituiría un obstáculo para el desarrollo normal de la vida familiar. La razón última de este planteamiento radicaba en que era el menor de cinco hermanos, todos ellos en edad infantil, carentes de cualquier pauta coherente de comportamiento y extraños a una dinámica familiar mínimamente rigurosa y estable.

En nuestras sucesivas visitas intentamos ayudar al niño a gatear y a dar sus primeros pasos, lo cual hacía sentado e impulsándose mediante el recurso de apoyar las manos en el suelo y entre sus piernas. En suma, en dos años y medio de vida no había desarrollado sus extremidades inferiores que, como puso de manifiesto un informe médico, acusaban una evidente deformación debida a la negligencia de los padres. Este hecho, por otra parte, coincidía con otra serie de conductas inadecuadas paralelas, en ámbitos tan distintos como la higiene, la alimentación, la organización de la vida o la educación.

Hechos como éste constituyen una experiencia cotidiana cuyo en- 
tendimiento y valoración, juntamente con otras variables que puedan incidir en cada caso, ayudan a entender la clave última de muchos casos que debemos abordar desde el Trabajo Social. El aprendizaje del niño y su proceso de personalización tiene lugar inicialmente en el plano socio-familiar, para ser luego una labor o tarea de carácter más individual. En este sentido, la atención al ambiente familiar, a los condicionamientos sociales, a los patrones culturales y, en suma, al contexto real en que se desenvuelve su vida, constituye una referencia inexcusable para cualquier intento de aproximación a sus problemas.

\section{LA INTERACCION NIÑO-ADULTO}

A propósito de la interacción niño-adulto, y entre otros posibles aspectos que podrían abordarse, hay que hacer alusión al hecho de que en muchos casos se insiste exclusivamente en que la familia de origen es la reproductora de las conductas desordenadas o deficientes. Es frecuente, por ejemplo, escuchar expresiones como «yo nunca he necesitado ir a la escuela», "nunca he ido al médico», "nunca he tenido que comer a la misma hora», etc..., que no son sino una manifestación verbal de la reproducción de conductas que han vivido anteriormente en la infancia y que, al estar plenamente interiorizadas, no son vistas como problemáticas sino que se consideran plenamente normales.

Pero el problema de base es más profundo. Porque la familia es el ámbito en que se inicia el proceso de socialización que va a condicionar de forma radical las conductas y los comportamientos del menor. En ella, va a ser testigo de situaciones de crisis y de cambio, de conflictos de diversa naturaleza, así como también de modos de intentar afrontarlos y resolverlos. Esta experiencia, conjuntamente con el trato de que sea objeto y el mayor o menor grado de protección y seguridad que viva van a configurar su propia autocomprensión como persona y su forma de interrelacionarse con otros en la época adulta.

El doctor Rof Carballo ha mantenido en España una atención constante al estudio de la incidencia que tienen los factores afectivos en la vida humana, destacando en particular el hecho de que «el estilo de cuidar al niño en los primeros meses y años de la vida repercute sobre sus condiciones de sociabilidad, su salud mental, su tendencia criminosa o su salud psico-física» ${ }^{1}$.

Consciente de que la tutela afectiva del niño actúa por modificaciones neurológicas y bioquímicas, es decir, estructurales, ha tenido empeño en subrayar que su conocido concepto de urdimbre, por el

1 Rof Carballo, J., Prólogo al volumen La Familia hoy. Convivencia y Conflicto, ASETES, Madrid, 1986, pág. 17. Una profundización de sus ideas puede verse, por ejemplo, en su? 
que expresa la estrecha vinculación que se establece impalpablemente entre el niño y los sercs responsables de su tutela, no responde sólo a una interpretación subjetiva sino que tiene una expresión que puede registrarse de forma objetiva. Esta es, entre otras, una de las razones por las que lamenta el hecho de que pocas veces se va al fondo del tema de la familia. En ella el hombre va formando estructuras biológicas que no llegan a su madurez sin el apoyo de la tutela amorosa, si no existe una atmósfera de amparo que haga posible la comunicación gozosa con la realidad. Como síntesis de su planteamiento, considero significativo y aleccionador el texto siguiente: «En muchísimos casos, esa familia que debería ser promotora de felicidad es presión, aherrojamiento. Poderosas fidelidades secretas a normas muchas veces estúpidas, se van transmitiendo de una generación a otra. Las llamadas fidelidades ocultas que a lo largo de generaciones pueden convertir a un niño, que debía ser fecundo habitante del universo, en un neurótico desgraciado o en un candidato a la locura y la delincuencia» ${ }^{2}$.

Los trastornos emocionales que provoca en el niño un ambiente familiar poco acogedor o deficientemente cohesionado se agudizan, sin duda, cuando en él se viven situaciones de conflicto o tensión que degeneran en la agresión o la violencia y el niño queda expuesto a los malos tratos que se derivan generalmente de ellas.

En este sentido, y como ilustración orientadora, considero que tiene un relieve importante el trabajo de síntesis publicado recientemente por P. Frontera ${ }^{3}$. Teniendo en cuenta el amplio espectro de tipos de malos tratos infantiles, propone el siguiente esquema de clasificación:

MALOS TRATOS INFANTILES

FISICOS PSIQUICOS SEXUALES

Lesiones no accidentales

Abuso emocional Incesto

Activos

Intoxicaciones y

Violación envenenamientos no accidentales

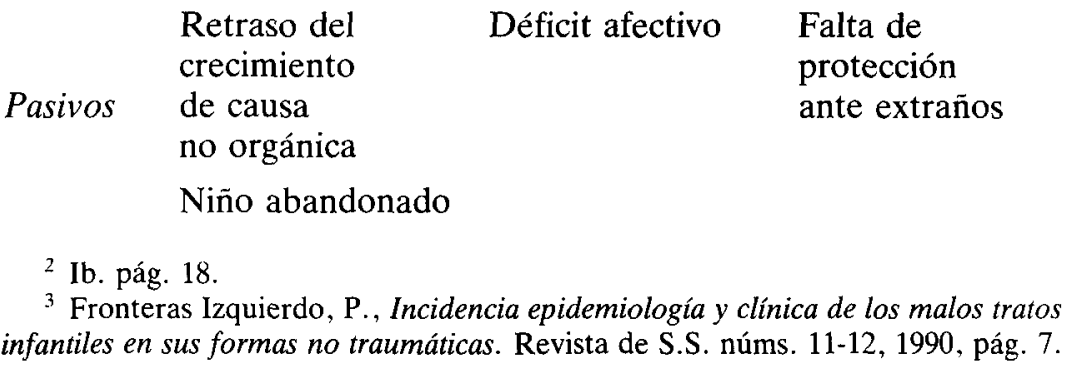


Además del comentario matizado de esta clasificación, nos interesa subrayar la actualidad de algunas consideraciones que allí se hacen en relación con el estudio de casos concretos, de los factores etiológicos de los malos tratos, de la intervención médico-social y de la valoración de los factores de riesgo. A propósito de estos últimos, se subraya la importancia especial que tienen los siguientes:

- Violencia intrafamiliar actual o anamnésica.

- Padre o madre maltratados o abandonados en su infancia.

- Pobreza, paro, grave problema económico.

- Ausencia de abuela «protectora» o presencia de un padrastro o madrastra hostil, cohabitando con el niño.

- Abandono de la madre, separación, o madre soltera rechazada por la familia.

- Enfermedad mental o adicción a alcohol o drogas.

- Padres indiferentes, intolerantes o superansiosos.

- Ausencia de lazos positivos madre-hijo.

- Niño prematuro, con déficit o con enfermedad (anticuerpos de SIDA).

- Madre menor de 18 años.

- Separación madre-hijo tras el parto ${ }^{4}$.

\section{LA INTERVENCION}

Volviendo ahora a nuestro planteamiento inicial, hay que resaltar el hecho de que la familia constituye el medio básico de socialización y de integración en la comunidad. Una familia marginada, si no es objeto de una intervención eficaz, da lugar a sucesivas generaciones de personas desadaptadas y a la reproducción de conductas y comportamientos que expresan claramente las características básicas de esa situación. En muchas ocasiones es patente la voluntad, explícita o no, de mantener las pautas de acción y los modos de interrelacionarse como una garantía de supervivencia frente a esquemas culturales o formas de convivencia a los que no se sienten capaces de adaptarse.

En orden a la programación y determinación de las características que ha de tener nuestra intervención en casos de esta naturaleza, entendemos que es útil distinguir dos tipos básicos de familia-problema:

- Familias plásticas: son aquellas que han ido amoldándose a las distintas situaciones de cambio que han debido afrontar a causa de problemas o crisis que las han llevado a adoptar algún tipo de conducta desadaptada. En estos casos es evidente la necesidad de ofrecerles recursos que faciliten su autonomía y capacidad de adaptación, colaborando con ellas en la búsqueda de alternativas. Se trata de familias que no plantean dificultades es-

${ }^{4}$ Ib. pág. 14. 
peciales para nuestro trabajo profesional, teniendo en cuenta la naturaleza específica de su problema.

- Familias rigidas: más estáticas y estereotipadas, que han ido heredando conductas inadecuadas y las han interiorizado y hecho propias como normales, sin ningún tipo de cuestionamiento. Todos los miembros de esas familias tienen unos comportamientos similares y unas pautas de acción prácticamente idénticas, por lo que es muy difícil que un miembro pueda salir de una situación-problema sin un apoyo especial exterior.

Todo ello hace que el profesional encuentre dificultades a la hora de intervenir en estas familias. Una ayuda para su trabajo podría ser la elaboración de un estudio diacrónico de una familia determinada, analizando los condicionamientos fundamentales de su desenvolvimiento a lo largo del tiempo (formas de vida, sistema de relaciones, rupturas entre generaciones, recursos económicos, etc.), así como los posibles apoyos institucionales y las características propias de la comunidad en que está inserta. Este conocimiento del entorno tiene una relevancia singular en este tipo de familias. En algunos casos, puede depender de él la supervivencia de las mismas o la posibilidad de un encauzamiento positivo de sus comportamientos. De ahí el interés que tiene contribuir a hacer posible una toma de conciencia de su situación real y de los modos de hacer frente a los retos que plantea su futuro, en orden a superar los condicionamientos y obstáculos que se oponen a la eliminación de su estado de dependencia, de marginalidad o de amenaza de desintegración.

En efecto, la influencia del entorno, entendido en un sentido amplio, tiene una singular relevancia en este tipo de familias, pudiendo depender de él incluso su propia supervivencia. Es, pues, indispensable intentar ayudarlas a tomar conciencia de las posibilidades reales del entorno, teniendo en cuenta que es muy limitado el grado de autonomía de que disponen para generar sus propios recursos e ir independizándose progresivamente. Puede ser ilustrativo el esquema siguiente:

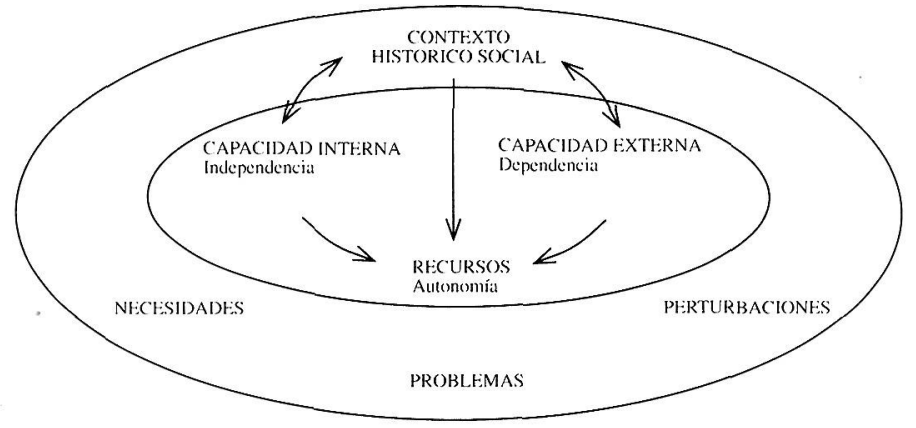


La buena utilización del contexto y el uso adecuado de recursos internos y externos determinarán, ante todo, la autonomía de la familia para salir de situaciones marginales o problemáticas. Incluso harán posible evitar que entre en ellas.

\section{TRANSMISION DISFUNCIONAL DEL ROL}

En términos generales, el rol que se desempeña con respecto a los hijos es de vital importancia. En el análisis y estudio de otras culturas, se puede observar que los roles que desempeñan los padres son diferentes. Por ejemplo, podemos establecer una comparación entre la cultura ashanti, en la que domina la descendencia matrilineal, con la cultura europea. En la primera, el padre es el encargado de la crianza de los hijos, de su alimentación, de la transmisión del oficio, mientras que la madre se ocupa de los asuntos sociales importantes, como son la herencia de las tierras, la sucesión en cargos y la política; es normal que se dé más valor a los lazos que vinculan con los hermanos que con el propio marido.

Es ilustrativa, en este sentido, la descripción siguiente que hace Hilda Kuper, y que transcribimos textualmente: «entre los swazi, la descendencia, la herencia, y la sucesión en el cargo siguen la línea paterna, pero las mujeres ocupan un puesto preeminente en la organización de la vida doméstica. La casa familiar está formada por un grupo de chozas dispuestas en semicírculo alrededor del corral del ganado y cada choza está ocupada por una madre y sus hijos. En el centro del arco está la gran choza ocupada por la madre del varón cabeza de familia (o por una de sus primeras esposas si su madre ha muerto). Cuando un joven o un grupo de hermanos funda una nueva casa la madre es instalada en el sitio de honor, porque ella fue la que los crió, defendió sus derechos, cuidó su patrimonio y los alimentó. El padre es respetado como jefe del grupo, reverenciado y, en su día, venerado como antepasado, pero la madre es amada y protegida» 5 .

«Esta situación es frecuente en las sociedades patriarcales, donde el padre es una figura autoritaria y dueño de los bienes que el heredero necesita para llegar a la plena madurez, lo que introduce una tensión latente en unas relaciones formalmente respetuosas. Parsons ha señalado que el rol de padre es siempre «instrumental», mientras que el de la madre tiene un carácter más emocional o expresivo (...).

En las sociedades tribales en que el parentesco es la base para la distribución de todos los roles sociales o de los más importantes, la familia ha de estar directamente articulada con los grupos de descenden-

${ }^{5}$ Radcliffe-Brown, A. R. y Forde, C., African Systems of Kinship and Marriage. Oxford Univ. Press, 1950. 
cia y se convierte en un mecanismo para la generación continua de nuevos lazos de parentesco. Como dice Fortes, "el taller, por así decirlo de la reproducción social es el grupo doméstico." ${ }^{6}$.

Otro aspecto a tener en cuenta es que, dentro de una misma sociedad, las diferentes subculturas también deben ser objeto de observación, al igual que las conductas patológicas que nos ocupan.

$\mathrm{Si}$ observamos las diferentes culturas, la familia ayuda a los hijos a formar parte de las mismas, reproduciendo los roles de los padres. La desorganización y la disolución de la familia perturba el proceso de socialización. En nuestra sociedad actual podemos comprobar cómo las familias en situación patológica proceden en su mayoría de hogares con los mismos síntomas; por ejemplo, las personas divorciadas tienen predisposición al divorcio. En suma, la falta de organización interna o la disolución vivida en la familia de origen repercute en el sentir de sus descendientes durante generaciones.

Goode define la desorganización en la familia como «el fraccionamiento de la unidad familiar, la disolución o quiebra de una estructura de roles sociales cuando uno o varios miembros no desempeñan adecuadamente las obligaciones propias de su rol» ${ }^{7}$.

Una vez observada la importancia del rol y su transmisión de generación en generación, pensamos que sería de gran utilidad hacer una clasificación de los elementos que influyen en la patología del mismo. Algunos de ellos tienen una relación estrecha entre sí, e incluso son consecuencia unos de otros, pero entendemos que es conveniente hacer referencia explícita de cada uno de ellos. Son éstos:

- Ausencia.

- Inmadurez.

- Abandono.

- Falta de afectividad.

- Autoestima y autopromoción.

- Imposibilidad física, cultural y emocional.

- Falta de protección.

- Falta de cooperación.

\section{Ausencia}

La ausencia del rol a desempeñar puede traer consigo una personalidad insegura, incomunicación, relaciones inestables, malos tratos, toxicomanías, prostitución, prejuicios culturales, desinterés personal y social, bajo nivel cultural, desviaciones sexuales, hacinamiento, pro-

${ }^{6}$ Godoy, J., The Developmental Cycle in domestic Groups. Cambridge Papers in Social Antropology, núm. 1, Cambridge Univ. Press.

${ }^{7}$ Goode, W. J., The family. Englewood Cliffs, N.J.: Ptrentice-Hall, 1964. 
miscuidad, falta de identidad, patrones rígidos de comportamiento, individualismo, valores desordenados.

\section{Inmadurez}

A la inmadurez se vinculan, de una forma $\mathbf{u}$ otra, la falta de discernimiento en la toma de decisiones, la incapacidad para mantener relaciones estables, la dificultad para elegir pareja y adaptarse a la vida familiar, la propensión a ceder fácilmente a las sugestiones de la drogadicción o prostitución, la atención mínima o superficial a temas o hechos de importancia social, la precocidad en relaciones sexuales o la desviación de las mismas, la falta de una visión previsora, la despreocupación por ingresos económicos, el hacinamiento, la promiscuidad, los malos tratos, la rigidez e intolerancia en las relaciones, la falta de conciencia colectiva, el conflicto de valores, la distorsión de la realidad, las enfermedades contagiosas, las intoxicaciones innecesarias, la ausencia de aseo personal, el individualismo, los conflictos vecinales, la disgregación familiar, la ausencia de liderazgo y la organización, la poca participación.

\section{Abandono}

La influencia del abandono en la patología del rol se evidencia en hechos que son también efecto de otros elementos. Piénsese, por ejemplo, en éstos: relaciones familiares inestables y ocasionales, incomunicación, malos tratos (negligencia), inseguridad, desadaptación, alcoholismo, drogadicción, desviaciones sexuales, desnutrición, enfermedades y falta de prevención de las mismas, falta de ingresos económi$\cos$, falta de identificación, neutralidad afectiva, rigidez, inadaptación, prejuicios, individualismo, dogmatismo, resentimiento social, dependencia, desempleo, improductividad, ausencia de sanciones, indiferencia en la educación, imposiciones, disgregación familiar, falta de solidaridad, ausencia de liderazgo.

\section{Falta de afectividad}

Entendiendo la afectividad en sentido amplio, su carencia es una de las claves que explican situaciones como éstas: inestabilidad familiar y personal, unión precoz de la pareja, incomunicación, malos tratos, celos, inseguridad, toxicomanías, prostitución, frustración, incesto, violación, desnutrición, falta de prevención (tanto de salud como de higiene), falta de identidad individual y familiar, automarginación, 
problemas de convivencia, infanticidio, delincuencia, mendicidad, indiferencia e irresponsabilidad.

\section{Autoestima y autopromoción}

Si se da a estas palabras la significación negativa que aquí se contempla, son el origen de: celos, toxicomanías, prostitución, frustración, relaciones sexuales patológicas, incesto, promiscuidad, malos tratos, prejuicios, intolerancias, desinterés, falta de concienciación, indiferencia, individualismo, inseguridad, desadaptación familiar, falta de preparación, sobreprotección, falta de cuidados personales y de la vivien$\mathrm{da}$, hacinamiento, inhabitabilidad, falta de identidad, ignorancia, falta de comunicación tanto interna como externa, desintegración en el grupo y en la comunidad.

\section{Imposibilidad física, cultural y emocional}

Desde distintas perspectivas, son constatables los efectos en ámbitos como: inestabilidad en la convivencia, incomunicación, personalidad insegura, desadaptación, desviaciones sexuales, sobreprotección, desnutrición, vivienda en mal estado, castigo, malos tratos exagerados, ignorancia, inadaptación al cambio, individualismo, dependencia económica, desempleo, imposición en la elección de pareja, enfermedad, infecciones e intoxicaciones.

\section{Falta de protección}

Este elemento, en estrecha relación con otros de los ya enumerados, provoca: inestabilidad, poligamia, precipitación en la elección de la pareja, incomunicación, malos tratos, celos, inseguridad, toxicomanías, prostitución, prejuicios sexuales, violación, desnutrición, hacinamiento, promiscuidad, neutralidad afectiva, desarraigo cultural, dependencia económica, castigos exagerados, procreación exagerada, accidentes, convivencia con animales domésticos, poca participación y comunicación con la comunidad.

\section{Falta de cooperación}

Consecuencias evidentes de ello son, por ejemplo: disgregación familiar, toxicomanías, desadaptación, individualismo, indiferencia, falta de solidaridad social, falta de solidaridad vecinal y comunal, descuido de bienes comunes, escaso respeto a los otros y desorganización. 


\section{SUGERENCIAS PARA UNA ALTERNATIVA}

Tras el diagnóstico podemos observar que el denominador común en la transmisión de conductas en muchos casos está determinado por la distorsión en los roles familiares y que la etiología de los problemas reside en muchos casos en la familia de origen tanto es aspectos físicos como en los psíquicos y sociales. El tratamiento con estas familias pensamos que no se debe hacer de forma unidireccional, teniendo en cuenta únicamente al menor sino que ha de realizarse de forma interaccionada entre

padres

hijos

contexto social

El aislamiento sistemático del menor en centros es, en la mayoría de los casos, improductivo porque lo único que se hace es soslayar el problema y resguardar a nuestros clientes, en este caso el menor, de una realidad no enfrentada ni superada. Y lo único que esto generará es un sentimiento proteccionista respecto al menor. Esto no quiere decir que el nin̄o tenga que soportar situaciones de alto riesgo en su medio que harían que el tratamiento terapéutico no fuera productivo, teniendo en cuenta que hay situaciones de emergencia social en las cuales hay que intervenir rápidamente.

Ultimamente se vienen gestionando recursos, como el acogimiento familiar, que en algunos casos puede ser una solución muy adecuada, sobre todo cuando nos encontramos ante situaciones como la anteriormente mencionada. Pero este recurso es mucho más fácil que la alternativa a que haremos referencia ahora.

Esta alternativa iría encaminada a que el niño y la familia intentaran modificar el contexto social adverso en el que viven, por medio de un equipo multiprofesional que les ayude en esa labor. Entendemos que es mucho menos costoso y más efectivo trabajar con un equipo multiprofesional, en el contexto y con la familia, que la institucionalización; esto supondría mayor implicación profesional y oficial (estatal). Profesionalmente es un trabajo de más alto riesgo y oficialmente es mucho menos vistoso que la institucionalización. Intentando no caer en demagogias o en utopías, es lógico preguntarse ¿cuántas veces se hace un estudio exhaustivo (con el correspondiente seguimiento de la situación problemática?) ¿cuántas veces se llega a hacer un buen diagnóstico de la situación? ¿cuántas veces se pasa de una mera gestión del recurso fácil?, etc.

Es evidente, por otra parte, que una profundización más rigurosa en la alternativa propuesta exigiría una reflexión amplia sobre el ejer- 
cicio de nuestra profesión, una valoración crítica de la política social actual y un examen a fondo de las consecuencias que se derivan para nuestra labor de la situación de crisis económica. En cualquier caso, entendemos que no es un despropósito sugerir la posibilidad de que existen alternativas eficientes que, con menores costes reales, pueden contribuir a hacer más eficaz nuestra labor profesional en un ámbito tan complejo y problemático como es el que aquí hemos estudiado.

La clave de la alternativa que aquí se sugiere, y que será objeto de un desarrollo amplio en otros trabajos posteriores, radica sobre todo en la convivencia diaria con nuestros clientes en su medio propio, poniendo los medios necesarios para que más adelante no sea ya un medio hostil ni continúe siendo un obstáculo a la relación interpersonal positiva y liberadora. Afrontando el problema desde una perspectiva amplia y exigente, con una mentalidad no individualista, y teniendo en cuenta todos los factores básicos que inciden en él, nuestros clientes aparecerán como el potencial más rico y efectivo que tenemos para intentar transformar ese medio. Ello significará dar a la práctica del Trabajo Social el significado profundo, social y comunitario, que justifica su existencia y que da sentido a nuestro compromiso profesional $^{8}$.

${ }^{8}$ Como orientación para este nuevo planteamiento que nos proponemos desarrollar, véanse, entre otros, Wolfe, D. A., Child abuse implications for child development and Psychopathology, California, 1987; Oates, K., Child abuse and neglect: what happens eventually, Nueva York, 1986; Hutchinson, R. L., The effects of family structure on institutionalized children's self-concepts, Adolescence, vol. XXIV, núm. 94, 1989. 